\title{
Aplicación del modelo polinomial en la evaluación de la uniformidad de riego del aspersor autocompensado Nelson R10
}

\author{
Polynomial model application in the uniformity \\ irrigation evaluation of the Nelson R10 flow self- \\ compensating sprinkler
}

Fecha de recepción: 1 de julio de 2014

Fecha de aceptación: 15 de octubre de 2014

Fanny del Rocío Rodríguez-Jarama', Francisco Javier del Cioppo-Mostardt ${ }^{2}$

\section{Resumen}

Los métodos de evaluación de la uniformidad de riego estiman las condiciones hidráulicas de funcionamiento del sistema de riego y su influencia en la aplicación de láminas de agua, con base en las necesidades hídricas de un cultivo. El modelo de evaluación utilizado en la presente investigación fue el polinomial. El ensayo evaluó el funcionamiento del sistema de riego con 4 presiones de operación distintas, medidas a la salida de la válvula del módulo de riego, y por cada presión de operación se tomaron 16 datos de caudales, en 5 módulos de riego instalado con el aspersor autocompensado Nelson R10. Como resultado se obtuvo el coeficiente de uniformidad, el coeficiente de distribución, el área adecuadamente irrigada y el factor de adecuación del riego. Uno de los principales resultados fue la no existencia de diferencia significativa entre las presiones mientras esta se mantengan en el rango de autocompensación; por otro lado, en el coeficiente de distribución sí existen diferencias significativas entre los tratamientos, a pesar de que todos dan valores mayores al $90 \%$, en contraste con los porcentajes del área relativa adecuadamente regada, que no superan el $50 \%$ en ninguno de los tratamientos. Con lo anterior se pudo validar y verificar que el aspersor Nelson R10 autocompensado, una vez instalado en campo, cumple con las características técnicas de operación expuestas en el catálogo del fabricante, sin embargo, sí se debe manejar y controlar la lámina de riego aplicada.

Palabras clave: Aspersor autocompensado, Modelo polinomial, Coeficiente de Uniformidad, Riego, Lámina de agua.

1 M. Sc. Universidad Agraria del Ecuador (Guayaquil, Ecuador). frodriguez@uagraria.edu.ec.

2 M. Sc. Universidad Agraria del Ecuador (Guayaquil, Ecuador). 


\begin{abstract}
There are irrigation evaluating uniformity methods, where the hydraulic operating conditions of the irrigation system and its influence on the water requirements of a crop are estimated. The evaluation methodology used in this research was the polynomial model. The study evaluated the functioning of the irrigation system with 4 different operating pressures, which were measured at the irrigation valve module exit, for each operating pressure 16 flow data were collected on 5 irrigation modules using a Nelson R10 self-compensating sprinkler.

The results of the research were: the uniformity coefficient, the distribution coefficient, the properly irrigated area and the irrigation suitability factor. One of the main results was the absence of significant difference between the pressures while they are in the range of auto compensation. On the other hand the distribution coefficient shows that there are significant differences between treatments, although all give values greater than $90 \%$, in contrast to the relative percentages of a properly irrigated area that does not exceed $50 \%$ in any of the treatments. It was validated and verified that the self-compensating installed sprinkler Nelson R10 complies with the technical operating characteristics exposed in the catalog of the manufacturer (self-compensating pressures and uniformity), however, the irrigation depth applied must be managed and controlled.
\end{abstract}

Keywords: Self-compensating sprinkler, Polynomial Model, Uniformity Coeficient, Irrigation system, Water layer. 


\section{Introducción}

La creación demodelos matemáticos o de técnicas de evaluación y ajuste de la distribución del agua aplicada de riego estuvo estancada por muchos años, a pesar de que son importantes para el funcionamiento de los sistemas de riego presurizados. Da Silva et al. (1) declaran que los avances recientes, como el modelo no lineal para el análisis de rendimiento de riego, que presentan resultados de evaluación y ajuste del desempeño de riego, son estadísticamente superiores a los de los modelos principales No lineales utilizados normalmente.

La evaluación de uniformidad de riego depende del diseño y la gestión de sistemas de riego, y de varios factores del suelo y el clima (3). Esta evaluación está orientada a identificar errores de diseño, operación y manejo del equipo. Utilizando instrumentación relativamente simple, se puede tener información muy útil para evaluar la operación del sistema y determinar si las presiones son las más adecuadas o los flujos de los aspersores corresponden al diseño propuesto; por lo que pueden conseguir ahorrar agua, mano de obra y energía (4).

Según Rázuri (5), para evaluar un sistema de riego presurizado se deben obtener indicadores de diseño como: 1) Un indicador cualquiera de uniformidad (Coeficiente de Uniformidad de Christiansen o coeficiente de distribución); 2) Dos indicadores del manejo (área adecuadamente regada y eficiencia de aplicación del agua) y el factor de adecuación del riego, que es la relación entre el promedio de agua aplicada para alcanzar una determinada meta de área adecuadamente irrigada, es decir, el promedio de agua asociado a un determinado perfil de distribución evaluado.

Con respecto al primer punto, Christiansen (6) proporciona una fórmula para determinar y caracterizar la uniformidad de la distribución del agua por los aspersores, que Zúñiga (7) lo ratifica, como son el Coeficiente de Uniformidad de Christiansen (CUC) y el Coeficiente de Distribución (CUD). Además, Merriam y Keller (8) han propuesto valores permitidos de CUC para los diferentes métodos de riego. Por lo que Alfaro (9) declara que el CUC sirve para indicar si el agua aplicada al suelo se dis- tribuye uniformemente; si la uniformidad es baja existirá mayor riesgo de déficit de agua en algunas zonas y de filtración profunda en otras.

Además, Saldarriaga (10) nos dice que el coeficiente de uniformidad de riego es una magnitud que caracteriza todo sistema de riego, y su objetivo debe ser tratar de obtener la mayor uniformidad posible, de tal manera que todas las plantas del cultivo reciban la misma cantidad de agua y nutrientes necesarios para alcanzar su desarrollo y la producción óptimos, ratificando así que la cantidad de lámina de riego bruta óptima depende de la uniformidad de riego y de la relación entre el precio del producto y el costo del agua(3).

También Zúñiga (7) expresa que para lograr valores altos en la uniformidad es necesario un traslape significativo entre aspersores, y que esto encarece el sistema, por lo que solo se pueden obtener buenas uniformidades en cultivos altamente rentables, y que se puede considerar un coeficiente de uniformidad del $85 \%$.

Con respecto al segundo punto, la eficiencia de riego se suele entender como el porcentaje de agua bruta aplicada que es aprovechada para satisfacer las necesidades del cultivo y del lavado (11); es una relación que expresa las pérdidas que ocurren desde la fuente de agua hasta las plantas, generalmente se expresa en porcentaje (12).

Da Silva et al.(1)utilizan un nuevo indicador para la evaluación de los sistemas de riego, denominado área adecuadamente irrigada, que expresa la fracción del área regada con aplicaciones mayor o igual al promedio general del agua aplicada. Normalmente, al ajustar el perfil de agua aplicada en la función polinomial (13), la línea divisoria en el gráfico elaborado por Da Silva representa el valor promedio que divide los volúmenes aplicados en exceso y en deficiencia en exactamente dos partes iguales.

En definitiva, la evaluación de la gestión del agua de riego puede definirse como la observación sistemática, la documentación y la interpretación de actividades relacionadas con el regadío, con el objetivo de una continua mejora, la misma que 
se debe realizar en el instante en que finaliza la instalación, al principio de cada campaña de riego y cuando existan motivos para sospechar la existencia de cambios en la uniformidad o lámina de agua aplicada (14).

Por lo tanto, el objetivo de esta investigación es evaluarla uniformidad de riego por aspersión subfoliar, con el aspersor autocompensado Nelson $\mathrm{R} 10$, con el propósito de incrementar su eficiencia.

\section{Materiales y métodos}

El experimento se llevó a cabo en la Hacienda Chaparral, ubicada en el Recinto Cerecita, perteneciente al Cantón Guayaquil, Provincia del Guayas, a 21 m.s.n.m., con temperatura promedio $31.3^{\circ} \mathrm{C}$ y precipitación de $971 \mathrm{~mm}$ por año, con una superficie de 50 ha, propiedad del señor Carlos Nebel, en donde se cultiva plátano asociado con cacao.

Para la evaluación de la Uniformidad del sistema de riego se deben conocer las características de este y las condiciones hidráulicas en que se encuentran sus distintas partes y accesorios, incluyendo el sistema de bombeo:

Tabla I. Condiciones hidráulicas del sistema de riego instalado Hacienda Chaparral

\begin{tabular}{|l|l|}
\hline Superficie de riego & 50 has \\
\hline Espaciamiento entre hileras de riego & $9 \mathrm{~m}$ \\
\hline Espaciamiento entre aspersores & $9 \mathrm{~m}$ \\
\hline Tipo de aspersor & Rotator Nelson R10 \\
\hline Boquilla instalada & $1,25 \mathrm{FC}$ plato Dorado P8 15 \\
\hline Caudal del emisor & $1,25 \mathrm{GPM}$; 284 Iph \\
\hline $\begin{array}{l}\text { Presión de autocompensación del } \\
\text { emisor }\end{array}$ & 30 m.c.a. \\
\hline Ciclo de Riego & 1 día \\
\hline Eto (lunes a sábado) & $4,7 \mathrm{~mm} /$ día \\
\hline Lámina bruta de riego & $5,2 \mathrm{~mm} /$ día \\
\hline Horas de riego por turno & 1,5 horas \\
\hline Turnos por ciclo & 9 turnos \\
\hline Presión en la estación de bombeo & 80 psi; 52 m.c.a. \\
\hline $\begin{array}{l}\text { Caudal erogado en la estación de } \\
\text { bombeo }\end{array}$ & $1200 \mathrm{GPM} ; 190$ m3/h \\
\hline
\end{tabular}

Parámetros agronómicos. Los parámetros agronómicos determinados fueron: Capacidad de Campo,
Punto de Marchitez Permanente, Saturación y Porcentaje de agua disponible, utilizando el método propuesto por Saxtony Willey(16), que consiste en la utilización del programa Soil Water Characteristics Hydraulic Properties Calculator, con el cual se estimó la tensión del agua del suelo, la conductividad y la capacidad de retención de agua, basado en la textura del suelo, la materia orgánica.

La Densidad aparente del suelo se determinó por el método del cilindro:

- Se llevó al campo un recipiente de cilindro muestreador de suelo; se determinó su volumen y su peso, y se introdujo en el suelo con un suave golpeteo de un martillo.

- Luego de haber extraído la muestra se pesó nuevamente el cilindro, y se puso en la estufa durante 48 horas a $105^{\circ} \mathrm{C}$, para eliminar la humedad de la muestra.

- Luego se pesó nuevamente el cilindro con la muestra, y con la siguiente fórmula se determinó la densidad aparente (Da):

$$
\mathrm{Da}=\mathrm{Ms} / \mathrm{Vs}
$$

Da: Densidad aparente

Ms: Masa del suelo seco

Vs: Volumen de suelo

Indicadores de desempeño hidráulico del riego: los indicadores de desempeño para cada tratamiento estudiado en esta investigación se determinaron, mediante el modelo matemático propuesto por Silva et al.(13), en una hoja de cálculo; es el modelo polinomial de quinto grado para representar el perfil de distribución de láminas de agua recolectadas en relación con la fracción del área total irrigada, el cual desarrolló relaciones matemáticas para el cálculo de los parámetros de la uniformidad y la eficiencia de aplicación del agua, facilitando analizar el desempeño y simplificar su utilización con estrategias de manejo y control de láminas de riego; este modelo representa distintos perfiles de distribución de agua, utilizando un mayor número de parámetros de ajustes. En este modelo se determinan parámetros del polinomio, usando la técnica de los mínimos cuadrados, de- 
rivando la siguiente expresión matemática en el cálculo del agua colectada Xc:X :

Se utilizó la planilla de cálculo para facilitar el ingreso de la información de datos de caudales y visualización de los resultados de coeficiente de uniformidad de Christiansen, coeficiente de uniformidad de distribución, eficiencia de aplicación, área adecuadamente regada y factor de adecuación del riego.

Previo a la recolección de los caudales, se realizaron las siguientes actividades:

Se recorrieron las instalaciones de la hacienda, verificando el funcionamiento óptimo de los accesorios y partes del sistema de riego.

Se revisó el funcionamiento del grupo de bombeo del sistema de riego, verificando la presión que aporta al sistema, así como el caudal que eroga, verificándolo en la válvula volumétrica.

Se identificaron en campo los módulos de riego con su respectiva válvula, con la utilización de GPS; los módulos son los lugares en donde se ejecutarán los tratamientos cada vez que estos trabajen en los turnos correspondientes de riego.

Se instalaron pilotos reguladores de presión en las válvulas para manejar las diferentes presiones que se proponen como tratamiento.

En la evaluación del sistema de riego con los diferentes tratamientos presentados, se tomaron datos de caudal de los aspersores en los distintos módulos ya identificados. El procedimiento que se realizó para la toma de datos fue el siguiente:

Se dividió el módulo siguiendo el esquema según la propuesta de obtención de datos de (2)

Se escogieron 16 aspersores del módulo, como se indica en la Fig. 1.

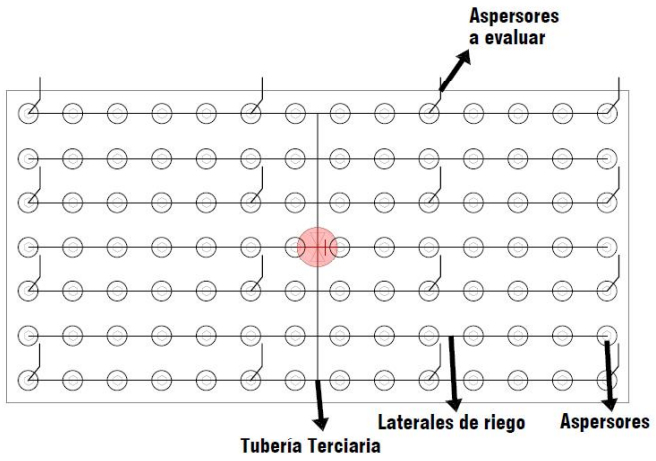

Figura 1. Modulo tipo del sistema de riego. Ubicación de aspersores evaluados.

Con el piloto regulador previamente instalado se reguló la presión en la válvula, según el tratamiento por evaluar; en cada aspersor se colocó una manguera y se recolectó el agua durante un minuto en un recipiente.

En cada uno de los aspersores se tomó el caudal, para cada una de las presiones correspondientes a los distintos tratamientos.

Luego se midió, con una probeta, la cantidad de agua en litros que se obtuvo en ese minuto.

Se registraron los datos de caudal en litros/min para cada aspersor por cada tratamiento y repetición en la hoja de toma de datos.

Diseño estadístico. Se utilizó el diseño de Bloques completamente al azar con 4 tratamientos y 5 repeticiones.

Tabla II. Tratamientos establecidos

\begin{tabular}{|l|c|c|}
\cline { 2 - 3 } \multicolumn{1}{c|}{} & $\begin{array}{c}\text { Presión } \\
\text { regulada en } \\
\text { la válvula }\end{array}$ & $\begin{array}{c}\text { Módulos o } \\
\text { repeticiones }\end{array}$ \\
\hline Tratamiento 1 & 15 m.c.a. & $5,19,30,53,64$ \\
\hline Tratamiento 2 & 20 m.c.a. & $5,19,30,53,64$ \\
\hline Tratamiento 3 & 25 m.c.a. & $5,19,30,53,64$ \\
\hline Tratamiento 4 & 30 m.c.a. & $5,19,30,53,64$ \\
\hline
\end{tabular}

m.c.a.: metros columnas de agua. 
La variabilidad entre los tratamientos se analizó mediante la prueba de Duncan al $5 \%$ de probabilidad.

Cada repetición es un módulo de riego; se evaluaron los módulos 5,19,30,53 y 64, de 69 que existían en el sistema de riego, escogiéndolos estratégicamente entre módulos cercanos, intermedios y lejanos a la bomba, en donde se tomaron datos de caudales que luego se procesaron en la plantilla antes mencionada.

Las variables analizadas fueron: Caudal, Coeficiente de Uniformidad, Coeficiente de Distribución, Eficiencia de aplicación, Área adecuadamente regada yFactor de adecuación del riego y la lámina.

\section{Resultados y discusión}

Parámetros agronómicos: con la utilización del calculador de propiedades hidráulicas SPAW, los resultados obtenidos fueron: capacidad de campo, $30,4 \%$; punto de marchitez permanente, 15,9\%; saturación, 46,4\%; porcentaje de agua disponible, 0,15 cm/cm; conductividad hidráulica, 10,85 $\mathrm{mm} / \mathrm{h}$, y densidad aparente, $1,42 \mathrm{~g} / \mathrm{cm}^{3}$.

Caudal en litros/minutos. Se tomaron 320 datos de caudales en el total del presente ensayo con los diferentes tratamientos, y según se indica en el análisis de varianza de los caudales en $\mathrm{I} / \mathrm{min}$ existe diferencia significativa entre los tratamientos. De acuerdo con la prueba de Duncan, al $5 \%$ de probabilidad el tratamiento 15 m.c.a. es el que tiene la media más baja, con $4,37 \mathrm{l} / \mathrm{min}$, y el tratamiento 30 m.c.a. es el de mayor caudal, con 4,73 l/min, a pesar de estar comprendido entre el rango de autocompensación.

Los tratamientos 20 m.c.a. y 25 m.c.a. no tienen diferencia significativa entre sí, y sus valores promedio de caudal están dados por $4,58 \mathrm{l} / \mathrm{min}$ y 4,63 I/min, respectivamente (ver Tabla III).

Coeficiente de uniformidad cuc: se obtuvieron 16 datos de caudal en litros/hora, recolectados en cada repetición (módulos de riego) para cada tratamiento (presiones), los cuales se procesaron en la plantilla para la evaluación del funcionamiento del sistema de riego, obteniendo valores de CUC para cada una de las interacciones, con los que se realizó el análisis de varianza.

Según el test de Duncan, no existen diferencias significativas entre los tratamientos estudiados respecto de la variable Coeficiente de Uniformidad (CUC); sin embargo, el promedio aritmético más alto lo obtuvo el tratamiento de 20 m.c.a., con $94,95 \%$, mientras que el tratamiento de 25 m.c.a. registró el promedio más bajo, con 94,33\%.

Coeficiente de distribución CUD: existen diferencias significativas entre los tratamientos estudiados respecto de la variable Coeficiente de Distribución (CUD). Los promedios aritméticos que se obtuvieron fueron: para el tratamiento 15 m.c.a., 91,77\%, y para el tratamiento 25 m.c.a., $91,85 \%$, que difieren estadísticamente de los tratamientos 20 m.c.a., cuyo promedio aritmético fue de $93,61 \%$, y 30 m.c.a., con $94,26 \%$.

Área relativa adecuadamente irrigada con el perfil originalmente modelado: los valores medios obtenidos del área adecuadamente irrigada con el perfil originalmente modelado fueron: para el tratamiento 15 m.c.a., 48\%; para el tratamiento 20 m.c.a., $36 \%$; para el tratamiento 25 m.c.a., $44 \%$, y para el tratamiento 30 m.c.a., $32 \%$. El tratamiento 30m.c.a. difiere significativamente de los demás por tener el promedio más bajo (32\%).

Se ha establecido que para los emisores Nelson R10 los coeficiente de uniformidad no registran diferencia significativa, manteniéndose constantes dentro de los rangos de presión evaluados, con valores mayores al $94 \%$ en todos los tratamiento; así como también el coeficiente de uniformidad de distribución, pues, aunque existen diferencias significativas entre los tratamientos, todos dan valores mayores al $90 \%$, lo que difiere de los porcentajes del área relativa adecuadamente regada, con valores que no superan el $50 \%$; esto demuestra que los datos de CUC y CUD no representan la efectividad con la que la lámina de agua está siendo aplicada en el área de riego.

Encontrar el área adecuadamente regada con el modelo propuesto por el Dr. Euzebio Medrado Da 
Silva (13) permitió representar claramente el déficit con que se están aplicando las láminas de riego en ciertas áreas; Kellery Bliesner (15) y Zúñiga (7) aseveran que el CUC y el CUD son parámetros que representan la distribución del agua de riego, pero aunque estos valores en la evaluación realizada fueron altos, Ilegando casi al $100 \%$, el área adecuadamente regada estuvo alrededor del $50 \%$.

Factor de adecuación del riego calculado con base en la selección del área adecuadamente irrigada: el factor que se debe adoptar para obtener una eficiente aplicación de riego del $90 \%$ es 1,0938 para el tratamiento 15 m.c.a., 1,0730 para el tratamiento 20 m.c.a., 1,0982 para el tratamiento 25 m.c.a. y 1,0616 para el tratamiento 30 m.c.a.; entre estos no existen diferencias. El tratamiento 30 m.c.a. es el de menor factor de adecuación por utilizar para ajustar la lámina de aplicación de riego $(1,0616)$.

Ajuste de la lámina aplicada con el factor de adecuación

De acuerdo con los resultados que se obtuvieron en los indicadores de desempeño hidráulico de riego, se procedió a ajustar la lámina de riego para cada factor de adecuación según los diferentes tratamientos, obteniendo así una nueva lámina de aplicación adecuada.

La lámina de diseño del sistema de riego de la Hacienda Chaparral es de 5,22 $\mathrm{mm} /$ día, pero al afectarlo con el factor de adecuación, la lámina del riego para el tratamiento 15 m.c.a. es 5,71 mm/día; para el tratamiento 20 m.c.a., 5,60mm/día; para el tratamiento 25 m.c.a., $5,73 \mathrm{~mm} /$ día, y para el tratamiento 30 m.c.a., la lámina de riego es 5,54 $\mathrm{mm} /$ día.

Eficiencia de aplicación final con el perfil de agua ajustado para la meta de adecuación del riego: según el test de Duncan, no existen diferencias significativas entre los tratamientos estudiados respecto de la variable eficiencia de aplicación; sin embargo, el promedio aritmético más alto lo obtuvo el tratamiento de 15 m.c.a., con 91,68\%, mientras que el tratamiento de 25 m.c.a. registró el promedio más bajo, con $86,23 \%$.

Tabla III. Promedio de las variables en los diferentes tratamientos

\begin{tabular}{|l|r|r|r|r|r|r|}
\hline TRATAMIENTO & $\begin{array}{c}\text { Caudal } \\
\text { (Itrs/min) }\end{array}$ & CUC \% & CUD \% & $\begin{array}{c}\text { AREA } \\
\text { ADECUADAMENTE } \\
\text { REGADA \% }\end{array}$ & $\begin{array}{c}\text { FACTOR DE } \\
\text { ADECUACIÓN }\end{array}$ & $\begin{array}{c}\text { EFICIENCIA DE } \\
\text { APLICACIÓN } \\
\text { FINAL \% }\end{array}$ \\
\hline Tratamiento 15 m.c.a & $4,37 \mathrm{~A}$ & $94,67 \mathrm{~A}$ & $91,77 \mathrm{~A}$ & $48 \mathrm{~B}$ & $1,0938 \mathrm{~B}$ & $91,21 \mathrm{~A}$ \\
\hline Tratamiento 20 m.c.a & $4,58 \mathrm{~B}$ & $94,95 \mathrm{~A}$ & $93,61 \mathrm{~B}$ & $36 \mathrm{~A}$ & $1,073 \mathrm{~B}$ & $92,97 \mathrm{AB}$ \\
\hline Tratamiento 25 m.c.a & $4,63 \mathrm{~B}$ & $94,33 \mathrm{~A}$ & $91,85 \mathrm{~A}$ & $44 \mathrm{~A}$ & $1,0982 \mathrm{~B}$ & $90,94 \mathrm{~A}$ \\
\hline Tratamiento 30 m.c.a & $4,73 \mathrm{C}$ & $94,41 \mathrm{~A}$ & $94,26 \mathrm{~B}$ & $32 \mathrm{~A}$ & $1,0616 \mathrm{~A}$ & $94,07 \mathrm{~B}$ \\
\hline Error experimental & 0,0041 & 1,926 & 1,166 & 0,77 & 0,0002 & 1,7347 \\
\hline
\end{tabular}

Letras distintas indican diferencias significativas $(p \leq 0,05)$.

\section{Conclusiones}

Con el modelo polinomial se representó el perfil de distribución de agua aplicada en el sistema de riego de la Hacienda Chaparral y se obtuvo un análisis que permitió observar que el tratamiento 20 m.c.a. tuvo mejor comportamiento en la entrega de láminas de agua, por lo cual es recomendable su uso para el manejo y control en sistemas presurizados.
Siendo el aspersor Nelson R10 un regulador de caudal en un rango depresión que va desde 20 m.c.a. hasta 32,5 m.c.a., se demuestra que con el tratamiento 15 m.c.a. hay diferencias estadísticas significativas, dando lugar a caudales menores que el caudal de operación del aspersor, pero también se concluye que el tratamiento 30 m.c.a. da diferencias significativas, obteniendo caudales mayores al de operación del aspersor a pesar de estar comprendido entre el rango de autocompensación. 


\section{Referencias}

(1) Da Silva E. M., Lima J. E. F. W., De Azevedo J. A., Rodrigues L. N. Proposição de um modelo matemático para a avaliação do desempenho de sistemas de irrigação. Pesquisa Agropecuária Brasileira. Vol. 39, n. ${ }^{\circ}$ 8, 2004.

(2) Del Cioppo M. y Proaño J. Análisis y manejo del riego a presión. Proyecto "Gestión del riego y la salinidad en los cultivos de la península de Santa Elena y la cuenca baja del río Guayas, Provincia del Guayas", Universidad Agraria del Ecuador, Embrapa (Brasil) y Programa de Apoyo Alimentario PL-480 - USDA. Guayaquil - Ecuador.2004.

(3) Mantovani, E.C. et al. Modelling the effects of sprinkler irrigation uniformity on crop yield. Agricultura Water Management. 1995.

(4) Llanes E, Milanes Y, Saavedra J. Análisis del sistema de riego "Ballama". Universidad de Granma, Cuba.2013.

(5) Rázuri L. Manejo y programación del riego. Programa de Maestría y Drenaje II. Universidad Agraria del Ecuador. Guayaquil - Ecuador.2012.

(6) Christiansen J.E. The uniformity of application of water by sprinkler systems. Agric. Eng.1941., 22: 89-92.

(7) Zúñiga É. Diseño y evaluación de riego a presión. San José Costa Rica. Editorial Universidad de Costa Rica.2004.

(8) Merriam J.L., and Keller I. Farm irrigation system evaluation: a guide for management. Utah State University, Logan, UT, 1978.271 pp.
(9) Alfaro A. Ahorro y eficiencia energética en agricultura de regadío. Riegos de Navarra S.A.2002.

(10) Saldarriaga J. Hidráulica de tuberías. Abastecimiento de agua, redes, riegos. Bogotá: Alfaomega, 2007; 690p.

(11) Montero J. Análisis de distribución de agua en sistemas de riego por aspersión estacionario: desarrollo del modelo de simulación de riego por aspersión, SIRIA: ediciones de la Universidad de Castilla La Mancha. 2000.

(12) García M. Eficiencia del riego. Tesis, Facultad de Agronomía Universidad de la República.2013.

(13) Da Silva E. et al. Proposta e seleção de uma função polinomia para a avaliação de sistemas de irrigação. Revista Brasileira de Engenharia Agrícola e Ambiental, vol.13, n. ${ }^{\circ 5}$, 2009; p.509-515.

(14) Junta de Regantes de Andalucía. Procedimiento para la realización de las evaluaciones de los riegos por aspersión. 2013. on line. disponible en: http://www.juntadeandalucia.es/ agriculturaypesca/ifapa/sar/contents/es/info/ infoSAR/protocolos/protocoloaspersion.pdf

(15) Keller J. and Bliesner R. D. Sprinkle and Trickle Irrigation. AVI Book. Van Nostrand Reinhold. New York.1990.

(16) Saxton K. E. and Willey P. H. The SPAW model for agricultural field and pond hydrologic simulation. Chapter 17. In: V. P. Singh and D. Frevert (Eds.), Mathematical Modeling of Watershed Hydrology.CRC Press LLC.2005. 